\title{
Histogram Thresholding Using Fuzzy and Rough Measures of Association Error
}

\author{
Debashis Sen, Member, IEEE, and Sankar K. Pal, Fellow, IEEE
}

\begin{abstract}
This paper presents a novel histogram thresholding methodology using fuzzy and rough set theories. The strength of the proposed methodology lies in the fact that it does not make any prior assumptions about the histogram unlike many existing techniques. For bilevel thresholding, every element of the histogram is associated with one of the two regions by comparing the corresponding errors of association. The regions are considered ambiguous in nature, and, hence, the error measures are based on the fuzziness or roughness of the regions. Multilevel thresholding is carried out using the proposed bilevel thresholding method in a tree structured algorithm. Segmentation, object/background separation, and edge extraction are performed using the proposed methodology. A quantitative index to evaluate image segmentation performance is also proposed using the median of absolute deviation from median measure, which is a robust estimator of scale. Extensive experimental results are given to demonstrate the effectiveness of the proposed methods in terms of both qualitative and quantitative measures.
\end{abstract}

Index Terms-Edge extraction, histogram thresholding, image segmentation, index of fuzziness, rough entropy, segmentation evaluation.

\section{INTRODUCTION}

$\mathbf{T}$ HRESHOLDING the histogram of an image, due to its simplicity and ease of implementation has been a popular technique used in various low-level image processing tasks. A plethora of bilevel histogram thresholding techniques exist in literature for purposes such as separating the foreground from the background in images [1]-[5] and removing the spurious edges during edge detection [6], [7]. Multilevel histogram thresholding finds application in partitioning an image into different regions [1], [8].

Comprehensive reviews of various histogram thresholding techniques are available in [9] and [10]. Most of these histogram thresholding algorithms are based on optimizing certain criteria, searching certain features such as "valleys" and "shoulders," or decomposing the histogram on the basis of modeling. However, as mentioned in [11], such methods would perform satisfactorily only when the histogram is "well-defined" with respect to the technique used, that is, when the histogram meets the prior assumptions made about it. Some examples

Manuscript received July 05, 2007; revised November 18, 2008. Current version published March 13, 2009. S. K. Pal was supported by the Government of India under the J. C. Bose National Fellowship. The associate editor coordinating the review of this manuscript and approving it for publication was Prof. Stanley J. Reeves.

The authors are with the Center for Soft Computing Research, Indian Statistical Institute, Kolkata, India 700108 (e-mail: dsen_t@isical.ac.in; sankar@isical.ac.in).

Digital Object Identifier 10.1109/TIP.2009.2012890 of histograms being "well-defined" are those which posses specific characteristics such as prominent "valleys" or "peaks," fit a particular model very well, or whose regions can be appropriately described using certain homogeneity measures. However, in practice, one can not guarantee that images having "well-defined" histograms will be encountered. Hence, it is desirable to have thresholding techniques that do not depend on whether the histogram is "well-defined" or not.

In this paper, we propose such a bilevel histogram thresholding method that assigns a bin of the histogram to one of the two classes based on the computation of certain association errors. The histogram of gray values in a grayscale image is considered here, unless mentioned otherwise. In the proposed methodology, the histogram is first divided into three regions, say, bright (a region of larger gray values), dark (a region of smaller gray values) and an undefined region. These regions are obtained using two predefined gray values, which are called the seed values. It is known (prior knowledge) that the bins of a histogram representing the smallest and the largest gray value would belong to the dark and bright regions, respectively. Hence, we consider that the graylevel bins of the histogram below the smaller seed value belong to the dark region and those above the larger seed value belong to the bright region. Rest of the graylevel bins form the undefined region. Then, each graylevel bin in the undefined region is associated with the defined regions, dark and bright, followed by the use of fuzzy (index of fuzziness [4]) or rough (rough entropy [12]) set theory to obtain measures of error due to the associations. The thresholding is then achieved by comparing the association errors and assigning each graylevel bin of the undefined region to one of the defined regions that corresponds to the lower association error. A similar technique that considers the similarity between graylevels for bilevel histogram thresholding has been proposed in [11]. However, we find that certain aspects of the method in [11] are flawed and, hence, might lead to incorrect thresholding (see Section III).

To carry out multilevel thresholding using the proposed scheme, more than two seed values would be required. Unlike bilevel thresholding, in the case of multilevel thresholding we do not posses the prior knowledge required to assign all the seed values. Hence, we present a tree structured technique that uses the proposed bilevel histogram thresholding scheme in order to carry out multilevel histogram thresholding. In this technique, each region (node) obtained at a particular depth are further separated using the proposed bilevel thresholding method to get the regions at the next higher depth. The required number of regions are obtained by proceeding to a sufficient depth and then discarding some regions at that depth using a certain criterion. 
The effectiveness of the proposed histogram thresholding methodology is demonstrated with the help of extensive experimental results and comparison with that of a few other existing algorithms. We apply the proposed and existing schemes for segmentation and edge extraction purposes, and especially use images with not "well-defined" histograms. The dependency of the proposed technique on the required input parameters, that is, the seed values, is also examined by carrying out statistical analysis.

We also present a new quantitative index to evaluate image segmentation performance and refer it as the VMAD-index. The proposed evaluation index measures variability using the median of absolute deviation from median (MAD) measure, which is a well-known outlier rejecting (robust) estimator [13].

In Section II, we present the measures of image ambiguity used in this paper. The explanation of the proposed bilevel and multilevel histogram thresholding methods are given in Sections III and IV, respectively. The proposed VMAD-index, various experimental results and comparisons, and parameter dependency analysis of the proposed methodology are presented in Section V. The paper concludes with Section VI.

\section{Measuring Image Ambiguity Using IndeX of FUZZINESS AND ROUGH ENTROPY}

Regions in a grayscale image do not have well-defined boundaries. Moreover, a gray value does not have appreciable discernibility from the nearby higher or lower gray values. These aspects of an image means that it is ambiguous in nature and so are the areas in an image corresponding to the dark, bright and undefined regions of the histogram. Ambiguity in images can be quantified using fuzzy and rough set theories [4], [12]. Therefore, as mentioned earlier, we consider fuzzy and rough set theories to measure certain association errors corresponding to regions in the histogram (see Section III). In this section, we briefly explain the measures of image ambiguity that have been used later in this paper.

\section{A. Ambiguity Measure Using Index of Fuzziness [4]}

Consider an $L$-level (graylevel) image $I$ and let $\Lambda$ be the universe of discourse of the $L$ gray values with the elements represented by $l_{i}, i=1,2, \ldots, L$. Let A be a set in $\Lambda$ which is given as $A=\left\{\left(l_{i}, \mu_{A}\left(l_{i}\right)\right)\right\}$. In order to define $A$, all the elements $l_{i}$ in $\Lambda$ are associated with values from the interval $[0,1]$, which gives the extent (membership) of possession of a particular property (that characterizes the set $A$ ) by these elements $\left(l_{i}\right)$. Such a set $A$ is called a fuzzy set and the corresponding values associated with $l_{i}$, which are denoted by $\mu_{A}\left(l_{i}\right)$, are referred to as membership grades. Here we consider that $\mu_{A}$ represents the brightness property [4] (defined on gray values) and use Zadeh's S-function [4] to calculate the membership values as follows:

$$
\begin{aligned}
\mu_{A}\left(l_{i}\right) & =S\left(l_{i} ; a, b, c\right), i=1,2, \ldots, L \\
\mu_{A}\left(l_{i}\right) & =0 \quad l_{i} \leq a \\
& =2\left[\frac{\left(l_{i}-a\right)}{(c-a)}\right]^{2} \quad a \leq l_{i} \leq b
\end{aligned}
$$

$$
\begin{aligned}
& =1-2\left[\frac{\left(l_{i}-c\right)}{(c-a)}\right]^{2} \quad b \leq l_{i} \leq c \\
& =1 \quad l_{i} \geq c
\end{aligned}
$$

where $a, b$, and $c$ are certain gray values, $b$ is referred to as the cross-over point given by $b=(a+c) / 2$ and a measure $\Delta b=b-a=c-b$ is called the bandwidth of the fuzzy set $A$. Index of fuzziness is a measure that can be used to quantify the average amount of fuzziness in the set $A$ and it is expressed as

$$
\nu_{p}=\frac{2}{(L)^{1 / p}}\left[\sum_{i=1}^{L}\left(\left|\mu_{A}\left(l_{i}\right)-\mu_{\underline{A}}\left(l_{i}\right)\right|\right)^{p}\right]^{1 / p}
$$

where $p$ is a positive integer and $\mu_{\underline{A}}\left(l_{i}\right)$ is given by

$$
\begin{aligned}
\mu_{\underline{A}}\left(l_{i}\right) & =0, & & \text { if } \mu_{A}\left(l_{i}\right) \leq 0.5 \\
& =1, & & \text { otherwise. }
\end{aligned}
$$

When the value of $p=1$, the measure is called the linear index of fuzziness and when $p=2$, it is called the quadratic index of fuzziness [4].

Now, let $X$ be a portion of $I$ having $N$ elements denoted by $x_{n}, n=1,2, \ldots, N$. Let $H_{X}\left(l_{i}\right)$ represent the number of elements in the array $X$ having the gray value $l_{i}$ (that is, $x_{n}=$ $l_{i}$ ). In the notation of fuzzy sets, $X$ may be considered as an array of fuzzy singletons represented as $X=\left[\left\{\left(x_{n}, \mu_{A}\left(x_{n}\right)\right)\right\}\right]$. Therefore, the expression of the index of fuzziness in (2) can be used to obtain a measure of ambiguity in $X$ as follows:

$$
\begin{aligned}
\nu_{p}^{X}=\frac{2}{(N)^{1 / p}}\left[\sum_{n=1}^{N}\left(\left|\mu_{A}\left(x_{n}\right)-\mu_{\underline{A}}\left(x_{n}\right)\right|\right)^{p}\right]^{1 / p} \\
=\frac{2}{(N)^{1 / p}}\left[\sum_{i=1}^{L}\left(\left|\mu_{A}\left(l_{i}\right)-\mu_{\underline{A}}\left(l_{i}\right)\right|\right)^{p}\right. \\
\left.\quad \times H_{X}\left(l_{i}\right)\right]^{1 / p} .
\end{aligned}
$$

Note that, the value of $\nu_{p}^{X}$ lies in the range $[0,1]$. Let us now consider a pictorial interpretation of the formula given in (3). Consider Fig. 1, where $H_{X}$ and $\mu_{A}$ are shown. As mentioned earlier, $\mu_{A}$ represents the brightness property, which means that the value of $\mu_{A}$ at each element gives the extent to which the element is bright. When the value of $\mu_{A}$ at an element is 0 or 1 , it is not ambiguous to the decide whether the element is bright or not bright. On the otherhand, when the value of $\mu_{A}$ at an element is greater than 0 and less than 1 , it is ambiguous to make such a decision about the element. In Fig. 1(a), the shaded region in $H_{X}$ represents the elements in $X$ which are associated with the aforesaid ambiguity. From the above explanation, it is straightforward that the formula in (3) gives an average measure of ambiguity in $X$.

\section{B. Ambiguity Measure Using Rough Entropy [12]}

Let us again consider an $L$-level (graylevel) image $I$ and let $\Lambda$ be the universe of discourse of the $L$ gray values with the elements represented by $l_{i}, i=1,2, \ldots, L$. We now consider the brightness property (defined on gray values) and define two 


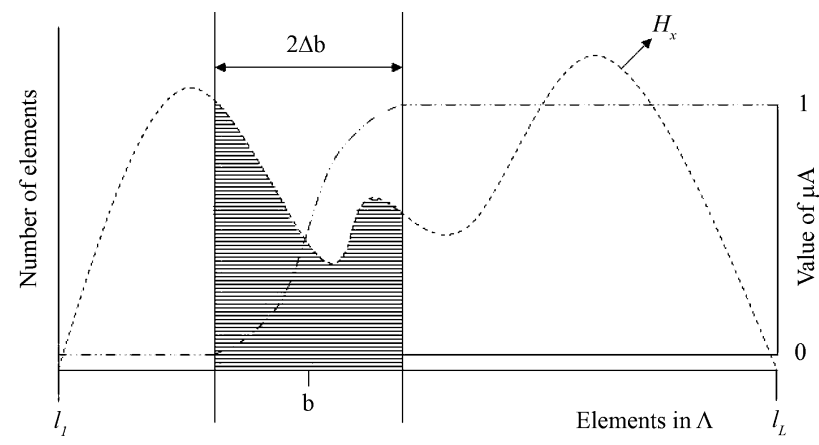

Fig. 1. Image ambiguity measurement using index of fuzziness.

mutually exclusive sets $A_{b}$ and $B_{b}$, respectively, representing the "bright" and "dark" gray values in $\Lambda$ as

$$
\begin{aligned}
& A_{b}=\left\{l_{i} \mid l_{i} \in \Lambda: l_{i}>b\right\} \\
& B_{b}=\left\{l_{i} \mid l_{i} \in \Lambda: l_{i} \leq b\right\} .
\end{aligned}
$$

As can be seen, $A_{b}$ and $B_{b}$ are defined such that $A_{b} \cup B_{b}=\Lambda$. The gray value $b$ is called the cross-over point. Now, as indiscernibility is created by the similarity between nearby gray values in the universe $\Lambda$, granules or induced equivalence classes (of equal size) are obtained by partitioning $\Lambda$. A granule in $\Lambda$ contains gray values which are considered similar to each other. As mentioned in [12], the granules are obtained such that the gray value $b$ is never at the boundary of a granule. Let us denote the size of the granules in $\Lambda$ by $\omega$. We consider the ambiguity in $\Lambda$ as the limited discernibility in its subsets created by the similarity between nearby gray values and then use rough set theory to obtain the lower approximations of $A_{b}$ and $B_{b}$, which are given as

$$
\begin{aligned}
& \underline{A}_{b}=\left\{l_{i} \mid l_{i} \in \Lambda:\left[l_{i}\right]_{\omega} \subseteq A_{b}\right\} \\
& \underline{B}_{b}=\left\{l_{i} \mid l_{i} \in \Lambda:\left[l_{i}\right]_{\omega} \subseteq B_{b}\right\}
\end{aligned}
$$

and the upper approximations of $A_{b}$ and $B_{b}$, which are expressed as

$$
\begin{aligned}
& \bar{A}_{b}=\left\{l_{i} \mid l_{i} \in \Lambda:\left[l_{i}\right]_{\omega} \cap A_{b} \neq \emptyset\right\} \\
& \bar{B}_{b}=\left\{l_{i} \mid l_{i} \in \Lambda:\left[l_{i}\right]_{\omega} \cap B_{b} \neq \emptyset\right\}
\end{aligned}
$$

where $\left[l_{i}\right]_{\omega}$ stands for granule of size $\omega$ containing the element $l_{i}$. The rough entropy measure [12] quantifying the ambiguity in the universe $\Lambda$ is given as

$$
R E_{b}(\Lambda)=-\frac{1}{2}\left[R_{A_{b}} \log _{e}\left(\frac{R_{A_{b}}}{e}\right)+R_{B_{b}} \log _{e}\left(\frac{R_{B_{b}}}{e}\right)\right]
$$

where

$$
R_{A_{b}}=1-\frac{\left|\underline{A}_{b}\right|}{\left|\bar{A}_{b}\right|}, \quad R_{B_{b}}=1-\frac{\left|\underline{B}_{b}\right|}{\left|\bar{B}_{b}\right|}
$$

In the above, $\left|\underline{A}_{b}\right|,\left|\bar{A}_{b}\right|,\left|\underline{B}_{b}\right|$, and $\left|\bar{B}_{b}\right|$ are the cardinalities of the sets $\underline{A}_{b}, \bar{A}_{b}, \underline{B}_{b}$, and $\bar{B}_{b}$, respectively.

Now, as considered earlier, let $X$ be a portion of $I$ having $N$ elements denoted by $x_{n}, n=1,2, \ldots, N$. Let $H_{X}\left(l_{i}\right)$ represent

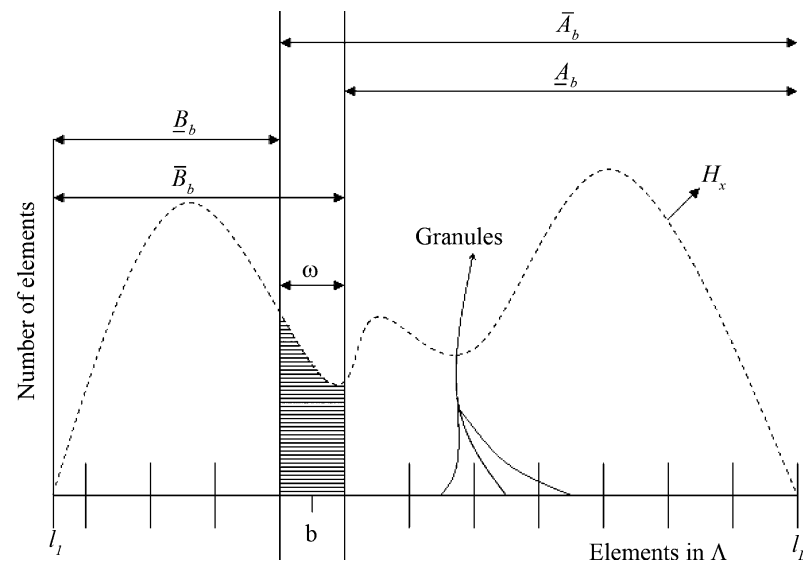

Fig. 2. Image ambiguity measurement using rough entropy.

the number of elements in the array $X$ having the gray value $l_{i}$ (that is, $x_{n}=l_{i}$ ). In order to find the rough entropy measure of ambiguity in $X$, we define

$$
R_{A_{b}}^{X}=1-\frac{\sum_{l_{i} \in \underline{A}_{b}} H_{X}\left(l_{i}\right)}{\sum_{l_{i} \in \bar{A}_{b}} H_{X}\left(l_{i}\right)}, R_{B_{b}}^{X}=1-\frac{\sum_{l_{i} \in \underline{B}_{b}} H_{X}\left(l_{i}\right)}{\sum_{l_{i} \in \bar{B}_{b}} H_{X}\left(l_{i}\right)} .
$$

We then obtain the expression for the rough entropy measure of ambiguity in $X$ as

$$
R E_{b}^{X}=-\frac{1}{2}\left[R_{A_{b}}^{X} \log _{e}\left(\frac{R_{A_{b}}^{X}}{e}\right)+R_{B_{b}}^{X} \log _{e}\left(\frac{R_{B_{b}}^{X}}{e}\right)\right] .
$$

Note that, the value of $R E_{b}^{X}$ lies in the range [0,1]. Let us now consider a pictorial interpretation of the formula given in (12). Consider Fig. 2, where $H_{X}, \underline{A}_{b}, \bar{A}_{b}, \underline{B}_{b}$, and $\bar{B}_{b}$ are shown. As mentioned earlier, the lower and upper approximations of $A_{b}$ and $B_{b}$ are considered as limited discernibility exists due to the similarity between nearby gray values in $\Lambda$. When an element belongs to the lower approximation of a set, it means that the element totally possesses the property characterizing the set (for example, every element in $\underline{A}_{b}$ is bright). When an element belongs to the lower approximation of the set of "bright" gray values $\left(\underline{A}_{b}\right)$ or to that of the set of "dark" gray values $\left(\underline{B}_{b}\right)$, it is not ambiguous to the decide whether the element is bright or dark. On the otherhand, when an element does not belong to both $\underline{A}_{b}$ and $\underline{B}_{b}$, it is ambiguous to make such a decision about the element. Therefore, we infer that the limited discernibility due to the similarity between nearby gray values in $\Lambda$ leads to the aforesaid ambiguity. In Fig. 2, the shaded region in $H_{X}$ represents the elements in $X$ which do not belong to both $\underline{A}_{b}$ and $\underline{B}_{b}$. From the above explanation, it is straightforward that the formula in (12) gives a measure of ambiguity in $X$.

\section{BileVel Histogram Thresholding BASED ON ASSOCIATION ERROR}

In this section, we propose a methodology to carry out bilevel histogram thresholding that does not make any prior 
assumption about the histogram. Let us consider two regions in the histogram of an image $I$ containing a few graylevel bins corresponding to the dark and bright areas of the image, respectively. These regions are obtained using two predefined gray values, say $g_{d}$ and $g_{b}$, with the graylevel bins in the range $\left[g_{b}, g_{\max }\right]$ representing the initial bright region and the graylevel bins in the range $\left[g_{\min }, g_{d}\right]$ representing the initial dark region. The symbols $g_{\min }$ and $g_{\max }$ represent the lowest and highest gray value of the image, respectively. A third region given by the graylevel bins in the range $\left(g_{d}, g_{b}\right)$ is referred to as the undefined region.

Now, let the association of a graylevel bin from the undefined region to the initial bright region causes an error of $E_{r r}$ units and the association of a graylevel bin from the undefined region to the initial dark region results in an error of $E r r_{d}$ units. Then, if $\operatorname{Err}_{d}>\operatorname{Err}_{b}\left(E_{r r}>E r r_{d}\right)$, it would be appropriate to assign the graylevel bin from the undefined region to the bright (dark) region. As boundaries in an image are not well-defined and nearby gray values are indiscernible, one can naturally consider the various areas in an image as ambiguous regions. Hence, the initial dark and bright regions of the histogram will represent two ambiguous areas in the image. Therefore, fuzzy and rough set theory, which have been extensively used to handle ambiguity, may be used to quantify the association errors.

\section{A. Proposed Methodology}

Here we present the methodology to calculate the error caused due to the association of a graylevel bin from the undefined region to a defined region. Using this method we shall obtain the association errors corresponding to the dark and bright regions, that is, $E r r_{d}$ and $E r r_{b}$. Each of these association errors comprise of two constituent error measure referred to as the proximity error and the change error.

Let $H_{i}$ represent the value of the $i$ th bin of the histogram of a grayscale image $I$. We may define $S_{b}$, the array of all the graylevel bins in the initial bright region as

$$
\begin{aligned}
S_{b} & =\left[H_{i}: i \in G_{b}\right], \text { where } \\
G_{b} & =\left[g_{b}, g_{b}+1, \ldots, g_{\max }\right]
\end{aligned}
$$

and $S_{d}$, the array of all the graylevel bins in the initial dark region as

$$
\begin{aligned}
S_{d} & =\left[H_{i}: i \in G_{d}\right], \text { where } \\
G_{d} & =\left[g_{\min }, \ldots, g_{d}-1, g_{d}\right]
\end{aligned}
$$

Now, consider that a graylevel bin from the undefined region corresponding to a gray value $g_{a}$ has been associated to the initial bright region. The bright region after the association is represented by an array $S_{b}^{a}$ as

$$
\begin{aligned}
S_{b}^{a} & =\left[H_{i}^{a}: i \in G_{b}^{a}\right], \text { where } \\
G_{b}^{a} & =\left[g_{a}, \ldots, g_{b}, \ldots, g_{\max }\right] \\
H_{i}^{a} & =H_{i} \text { when }\left(i=g_{a} \text { or } i \geq g_{b}\right) \\
H_{i}^{a} & =0 \text { elsewhere. }
\end{aligned}
$$

In a similar manner, the dark region after the association is represented by an array $S_{d}^{a}$ as

$$
\begin{aligned}
S_{d}^{a} & =\left[H_{i}^{a}: i \in G_{d}^{a}\right], \text { where } \\
G_{d}^{a} & =\left[g_{\min }, \ldots, g_{d}, \ldots, g_{a}\right] \\
H_{i}^{a} & =H_{i} \text { when }\left(i=g_{a} \text { or } i \leq g_{d}\right), \\
H_{i}^{a} & =0 \text { elsewhere. }
\end{aligned}
$$

In order to decide whether the graylevel bin corresponding to the gray value $g_{a}$ belongs to the bright or dark region, we need to determine the corresponding errors $E r r_{d}$ and $E r r_{b}$. As mentioned earlier, our measure of an association error $(E r r)$ comprises of a proximity error measure $\left(e_{p}\right)$ and a change error measure $\left(e_{c}\right)$. We represent an association error as

$$
\operatorname{Err}=\left(\alpha+\beta e_{c}\right)+e_{p}
$$

where $\alpha$ and $\beta$ are constants such that $\alpha+\beta e_{c}$ and $e_{p}$ take values from the same range, say, $[0,1]$.

In order to determine the errors $e_{p}$ and $e_{c}$ corresponding to the bright and dark regions, let us consider the arrays $S_{b}^{a}$ and $S_{d}^{a}$, respectively. We define the change error due to the association in the bright region as

$$
e_{c}^{b}=\frac{\operatorname{Amb}\left(S_{b}^{a}\right)-\operatorname{Amb}\left(S_{b}^{a}\right)}{\operatorname{Amb}\left(S_{b}^{a}\right)+\operatorname{Amb}\left(S_{b}^{a}\right)}
$$

where the array $S_{b}^{a}$ is obtained by replacing $H_{g_{a}}^{a}$ by 0 in $S_{b}^{a}$ and $\operatorname{Amb}\left(S_{\Omega}\right)$ gives the ambiguity in the image region represented by the graylevel bins in an array $S_{\Omega}$ as a real value in the range $[0,1]$. The function $A m b(\cdot)$ is implemented using fuzzy or rough set theory, which shall be explained later. Now, in a similar manner, the change error due to the association in the dark region is given as

$$
e_{c}^{d}=\frac{\operatorname{Amb}\left(S_{d}^{a}\right)-\operatorname{Amb}\left(S_{d}^{a}\right)}{\operatorname{Amb}\left(S_{d}^{a}\right)+\operatorname{Amb}\left(S_{d}^{a}\right)}
$$

where the array $S_{d}^{a}$ is obtained by replacing $H_{g_{a}}^{a}$ by 0 in $S_{d}^{a}$. It is evident that the expressions in (18) and (19) measure the change in ambiguity of the regions due to the association of $g_{a}$, and, hence, we refer the measures as the change errors. The form of these expressions is chosen so as to represent the measured change as the contrast in ambiguity, which is given by the ratio of difference in ambiguity to average ambiguity. As can be deduced from (18) and (19), the change errors would take values in the range $[-1,1]$. It is also evident from (18) and (19) that the change error may take a pathological value of $0 / 0$. In such a case, we consider the change error to be 1 .

Next, we define the proximity errors due to the associations in the bright and dark regions, respectively, as

$$
\text { and } \begin{aligned}
e_{p}^{b} & =1-\operatorname{Amb}\left(S_{b}^{a}\right) \\
e_{p}^{d} & =1-\mathrm{C} \times \operatorname{Amb}\left(S_{d}^{a}\right) .
\end{aligned}
$$

In the above, we take $e_{p}^{d}=0$, if $\mathrm{C} \times \operatorname{Amb}\left(S_{d}^{a}\right)>1$. It will be evident later from the explanation of the function $\mathrm{Amb}(\cdot)$, that the ambiguity measures in (20) and (21) increase with the increase in proximity of the graylevel bin corresponding to $g_{a}$ 


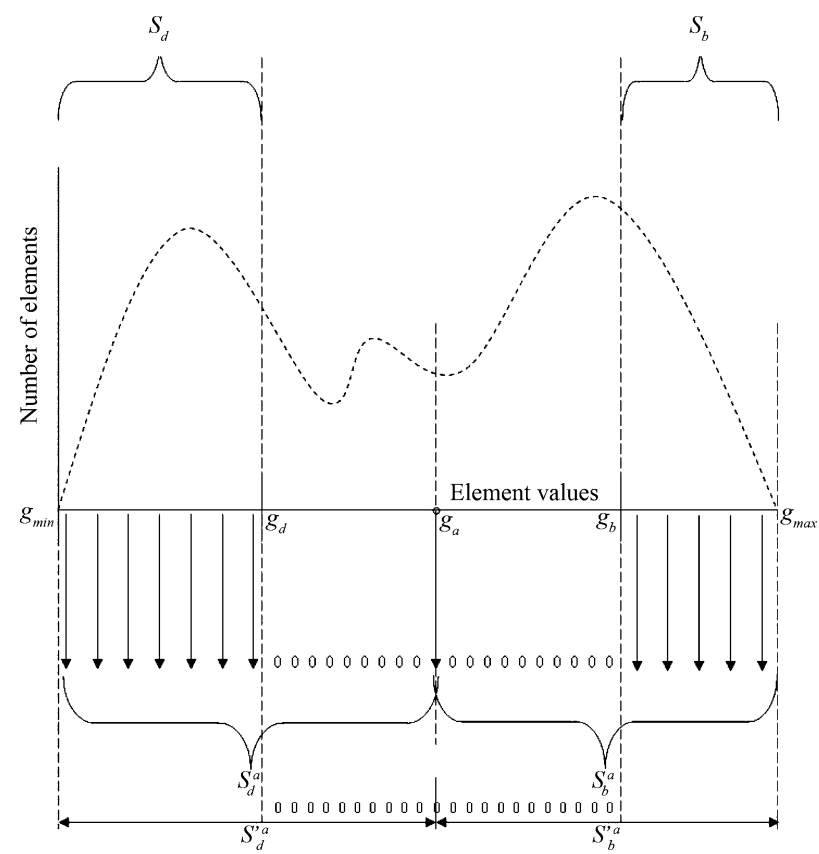

Fig. 3. Various defined arrays shown for a multimodal histogram.

from the corresponding regions. Thus, the expressions in (20) and (21) give measures of farness of the graylevel bin corresponding to $g_{a}$ from the regions, and, hence, we refer the measures as the proximity errors. The symbol $\mathrm{C}$ is a constant such that the values of $e_{p}^{b}$ and $e_{p}^{d}$ when $g_{a}$ equals $g_{b}-1$ and $g_{d}+1$, respectively, are the same and, hence, the proximity error values are not biased towards any region. As can be deduced from (20) and (21), the proximity errors would take values in the range $[0,1]$. The various arrays defined in this section are graphically shown in Fig. 3.

The implementation of function Amb $(\cdot)$ using Fuzzy Sets and Rough Sets: Let us first consider an array $S_{\Omega}$ which has the graylevel bins corresponding to the gray values given in another array say $G_{\Omega}$ and let the length of the arrays be $N_{\Omega}$. We shall now define an array $X_{i n}$ as follows:

$$
X_{\text {in }}=\left[\left[G_{\Omega}(1)\right]_{S_{\Omega}(1)}, \ldots,\left[G_{\Omega}\left(N_{\Omega}\right)\right]_{S_{\Omega}\left(N_{\Omega}\right)}\right]
$$

where a notation $\left[G_{\Omega}(1)\right]_{S_{\Omega}(1)}$ represents an array formed by repeating the value $G_{\Omega}(1), S_{\Omega}(1)$ number of times. Considering the array $X_{i n}$ as the array $X$ (portion of an image) mentioned in Section II, the index of fuzziness and rough entropy based measures of ambiguity in $X_{i n}$ can be calculated. One of these measures is considered as the output of the function $\mathrm{Amb}(\cdot)$ applied to the array $S_{\Omega}$ and represented as $\operatorname{Amb}\left(S_{\Omega}\right)$. Note that arrays such as $X_{i n}$ can be obtained for the arrays $S_{o}^{a}, S_{b}^{a}, S_{o}^{a}$, and $S_{b}^{a}$ defined earlier, and, hence, the measures $\operatorname{Amb}\left(S_{o}^{a}\right), \operatorname{Amb}\left(S_{b}^{a}\right)$, $\operatorname{Amb}\left(S_{o}^{\prime}\right)$, and $\operatorname{Amb}\left(S_{b}^{a}\right)$ are given in a manner similar to the one explained above.

From Section II, we find that we need to define the cross-over point $b$, the bandwidth $\Delta b$ of the S-function and the granule size $(\omega)$ in order to calculate the index of fuzziness and rough entropy based ambiguity measures. For the calculation of the association errors corresponding to the bright and dark regions, we define the respective cross-over points as

$$
\begin{aligned}
b_{b} & =\frac{g_{a}+g_{b}}{2} \\
\text { and } \quad b_{d} & =\frac{g_{d}+g_{a}}{2} .
\end{aligned}
$$

Considering the above expressions for the cross-over points and the explanation in Section II, it can be easily deduced that the ambiguity measures in (20) and (21) increase with the increase in proximity of the graylevel bin corresponding to $g_{a}$ from the defined regions, as mentioned earlier.

While calculating the association errors corresponding to both the bright and dark regions, it is important that same S-function bandwidth $(\Delta b)$ and same granule size $(\omega)$ be considered. Although any constant value can be assigned to $\Delta b$ and $\omega$, as suggested in [4], we vary the values of $\Delta b$ and $\omega$ over a range. As presented earlier in (17), the errors due to the association of a gray value from the undefined region to the dark and the bright region are given as

$$
\begin{aligned}
& \operatorname{Err}_{d}=\left(\alpha+\beta e_{c}^{d}\right)+e_{p}^{d} \\
& \operatorname{Err}_{b}=\left(\alpha+\beta e_{c}^{b}\right)+e_{p}^{b} .
\end{aligned}
$$

We calculate the association errors $E r_{d}$ and $E r_{b}$ for all graylevel bins corresponding to $g_{a} \in\left(g_{d}, g_{b}\right)$, that is, the graylevel bins of the undefined region. We then compare the corresponding association errors and assign these graylevel bins to one of the two defined (dark and bright) regions that corresponds to the lower association error. In (25) and (26), we consider $\alpha=\beta=0.5$ and, hence, force the range of contribution from the change errors to $[0,1]$, same as that of the proximity errors. Thus, the bilevel thresholding is achieved by separating the bins of the histogram into two regions, namely, the dark and the bright regions.

As mentioned in Section I, a bilevel thresholding technique similar to the one proposed in this section has been reported in [11]. The authors in [11] have considered the indeces of fuzziness of the two defined regions after the association of a graylevel bin from the undefined region as measures of similarity and then compared these similarity measures (fuzziness) corresponding to the two defined regions in order to carry out the thresholding operation. Here, we point out two extremely important aspects of the algorithm in [11], which are flawed and might lead to incorrect thresholding.

1) It is appropriate to compare two fuzziness values only when they have been calculated using similar membership functions having the same bandwidth (same units). Although the algorithm given in [11] uses similar membership functions for calculating the fuzziness values, same bandwidth is not guaranteed.

2) Let us consider the error due to the association of a graylevel bin of certain value in the undefined region to the defined dark and bright regions be $E r r_{d}$ and $E r r_{b}$, respectively. Let the ambiguities in the initial dark and bright regions obtained using the seed values are equal. Then, if the proximity of the graylevel bin under consideration from the bright region is greater than that from the 
dark region then $\operatorname{Err}_{b}$ must be less than $\operatorname{Err}_{d}$ and vice versa. The algorithm given in [11] does not guarantee such a condition.

The above problems do not exist in the proposed methodology.

\section{MultileVel Histogram Thresholding BASED ON ASSOCIATION ERROR}

In this section, we extend the bilevel histogram thresholding methodology proposed in the previous section to the multilevel histogram thresholding problem. One way of carrying out this extension would be to formulate a function of the gray values in the undefined region based on their $E r r_{d}$ and $E_{r r}$ measures and then search for a predefined number of local optima in the function and consider them as the required thresholds. But, this approach would have the following drawbacks.

1) Presence of a predefined number of local optima would not be guaranteed and, hence, such an algorithm may not always be feasible.

2) As mentioned above, the function of the gray values would be based on $E r r_{d}$ and $E r_{b}$ measures. Note that these measures are obtained using two seed values representing the initial dark and bright regions. Thus, as the function would relate to the association errors obtained with respect to two initially defined regions, it is inappropriate to get multiple regions (multilevel thresholding) in the histogram based on such a function. In order to carry out multilevel thresholding, multiple seed values should be used.

As mentioned in Section I, we do not posses the prior knowledge required to assign more than two seed values. Therefore, we understand that the concept of thresholding based on association error can be used to separate a histogram only into two regions and then these regions can further be separated only into two regions each and so on. From this understanding, we find that the proposed concept of thresholding using association error could be used in a tree structured technique in order to carry out multilevel thresholding.

Now, let us consider that we require a multilevel histogram thresholding technique using association error in order to separate a histogram into $\Theta$ regions. Let $D$ be a non-negative integer such that $2^{D-1}<\Theta \leq 2^{D}$. In our approach to multilevel histogram thresholding for obtaining $\Theta$ regions, we first separate the histogram into $2^{D}$ regions. The implementation of this approach can be achieved using a binary tree structured algorithm [14]. Note that in [14], the binary tree structure has been used for classification purposes, which is not our concern. In our case, we use the binary tree structure to achieve multilevel histogram thresholding using association error, which is a totally unsupervised technique. We list a few characteristics of a binary tree below stating what they represent when used for association error based multilevel histogram thresholding.

1) A node of the binary tree would represent a region in the histogram.

2) The root node of the tree represents the histogram of the whole image.

3) The depth of a node is given by $D$. At any depth $D$ we always have $2^{D}$ nodes (regions).

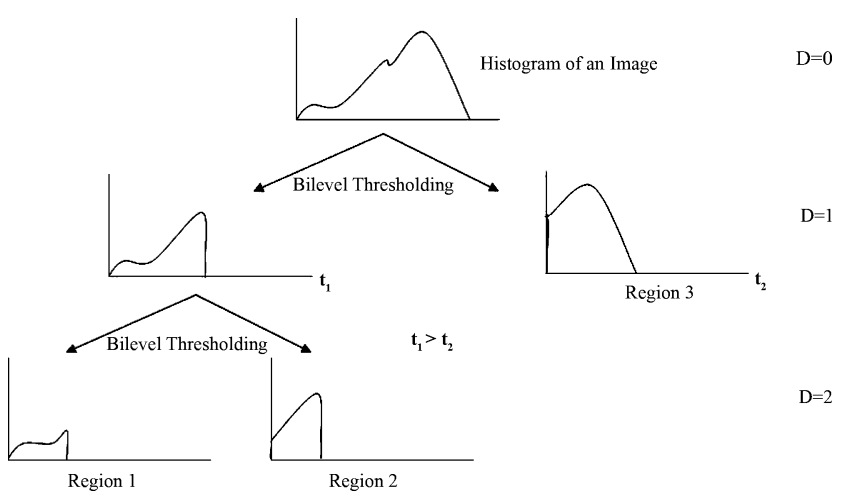

Fig. 4. Separation of a histogram into three regions using the proposed multilevel thresholding based on association error.

4) Splitting at each node is performed using the bilevel histogram thresholding technique using association error proposed in the previous section.

5) All the nodes at a depth $D$ are terminal nodes when our goal is to obtain $2^{D}$ regions in the histogram.

In order to get $\Theta$ regions from the $2^{D}$ regions, we need to declare certain bilevel thresholding of histogram regions (node) at depth $D-1$ as invalid. In order to do so, we define a measure $(\iota)$ of a histogram region based on the association errors $E r r_{d}$ and $E r r_{b}$ obtained for the values of $g_{a}$ (see Section III) corresponding to the histogram region as follows:

$$
\iota=\sum_{g_{a} \in\left(g_{d}, g_{b}\right)} \operatorname{Err}_{d}\left(g_{a}\right)+\operatorname{Err}_{b}\left(g_{a}\right)
$$

where $g_{a}, g_{d}$ and $g_{b}$ are the same as explained in the previous section, except for the fact that they are defined for the underlying histogram region and not for the entire histogram. We use the expression in (27) to measure the suitability of the application of the bilevel histogram thresholding technique to all the histogram regions at the depth $D-1$. Larger the value of $\iota$ for a region of the histogram, more is the corresponding average association error and, hence, more is the suitability. Hence, in order to get $\Theta$ regions, we declare the bilevel thresholding of $2^{D}-\Theta$ least suitable (based on $\iota$ ) regions at depth $D-1$ as invalid, and, hence, we are left with $\Theta$ regions at depth $D$. Fig. 4 graphically demonstrates the use of proposed multilevel thresholding technique using association error in order to obtain three regions (Regions 1, 2, and 3) in the histogram. The values $\iota_{1}$ and $\iota_{2}$ gives the suitability of the application of the bilevel thresholding on the two regions at depth $D=1$.

\section{EXPERIMENTAL RESULTS AND COMPARISONS}

In this section, we provide experimental results evaluating the performance of the proposed bilevel and multilevel histogram thresholding methods and compare them with that of a few existing algorithms. We use the proposed and other thresholding techniques to carry out segmentation, object/background separation and edge extraction in images.

\section{A. Segmentation and Object/Background Separation}

The use of the proposed thresholding methodology in performing segmentation and object/background separation in im- 


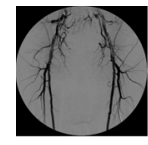

(a)

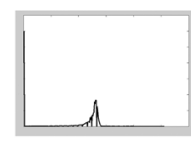

(b)

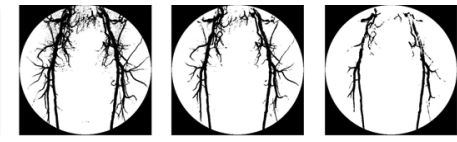

(c) (d) (e)

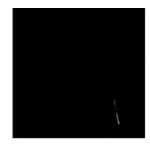

(f)

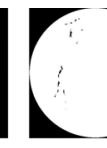

(g)

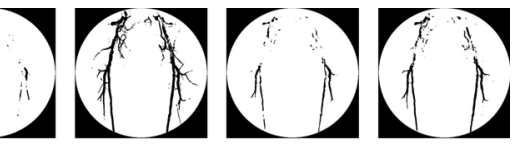

(i) (j)

Fig. 5. Qualitative results obtained using the various thresholding algorithms applied to extract blood vessels in an angiography image. (a) Image. (b) Histogram. (c) Seg. by (i). (d) Seg. by (ii). (e) Seg. by (iii). (f) Seg. by (iv). (g) Seg. by (v). (h) Seg. by (vi). (i) Seg. by (vii). (j) Seg. by (viii).

ages is presented here. Both quantitative and qualitative evaluation of performance are considered for study and comparisons. Quantitative evaluation methods can be classified into those which require ground truth and those which do not. Segmentation results can be evaluated by homogeneity based measures such as the $\beta$-index [15] which do not require ground truth. The expression for $\beta$-index is

$$
\beta=\frac{\sum_{i=1}^{c} \sum_{j=1}^{n_{i}}\left(I_{i j}-\bar{I}\right)^{2}}{\sum_{i=1}^{c} \sum_{j=1}^{n_{i}}\left(I_{i j}-\bar{I}_{i}\right)^{2}}
$$

where $c$ gives the number of regions obtained after the segmentation process, $n_{i}$ is the number of pixels in the $i$ th region, $I_{i j}$ is the gray value of the $j$ th pixel in the $i$ th region of an image $I, \bar{I}_{i}$ is the mean of the gray values in the $i$ th region and $\bar{I}$ is the mean of the gray values in the image $I$. In the case of evaluation of object/background separation in images we have $c=2$.

Note that $\beta$-index uses sum of squared distance from mean as the underlying measure of variability. It is well-known that such a measure of variability does not reject extreme values or outliers effectively. Hence, the presence of a very few extreme values of variation could have a greater effect on the $\beta$-index measure than the significant majority of moderate values of variation. We suggest that a segmentation evaluation index should ignore the effect of an insignificant minority of extreme values of variation (unlike $\beta$-index), and, hence, we present an alternative segmentation evaluation index in this regard.

A segmentation evaluation measure-VMAD-index:

Here we propose a new homogeneity based index, which is less affected by extreme values of variation, to evaluate segmentation results quantitatively. We call this index as the VMADindex which calculates the ratio of the total variation in an image to the sum of within-region variations. We use the median of absolute deviation from median (MAD) measure [13], which is a well-known robust estimator of scale, in order to quantify the variations. Hence, the VMAD-index is not heavily influenced by a minority of extreme values of variation and it is given as

$$
\operatorname{VMAD}=\frac{\operatorname{Med}\left(\left|I_{I k}-\operatorname{Med}(I)\right|\right)}{\sum_{i=1}^{c}\left(\frac{n_{i}}{n_{I}}\right) \times \operatorname{Med}\left(\left|I_{i j}-\operatorname{Med}\left(I_{i}\right)\right|\right)}
$$

where $\operatorname{Med}(A)$ represents the median element value in the array $A$ and $I_{I k}$ stands for the gray value of the $k$ th pixel in the image $I$. The symbols $I_{i j}, c$ and $n_{i}$ are the same as the ones defined for (28), $n_{I}$ is the number of pixels in the image $I$ and $I_{i}$ represents the $i$ th region in the image $I$. It is evident from (29) that for an image, the VMAD-index increases with increase in homogeneity in the regions and, hence, higher the value of VMAD-index better the segmentation. The VMAD-index takes values in the range $[0, \infty]$ for a given value of $c(c$ is a integer with $1<c \leq L, L$ being the number of graylevels). Note that, in order to quantify the variations, one may also use the $S_{n}$ or $Q_{n}$ measure proposed in [16], which are also robust estimators of scale having certain advantages over the MAD measure. We shall use both $\beta$-index and VMAD-index while presenting the quantitative results of the proposed and other histogram thresholding techniques.

Let us first consider the visual assessment of segmentation results in different images in order to evaluate the qualitative performance of the various techniques. The techniques considered for comparison are: (i) thresholding based on fuzzy measure of association errors (proposed), ii) thresholding based on rough measure of association errors (proposed), (iii) Otsu's method [1], (iv) method by Kapur et al. [2], (v) method by Kittler $e t$ al. [3],(vi) Tsai's method [5], (vii) fuzzy graylevel similarity based thresholding [11], and (viii) thresholding based on index (linear) of fuzziness [4]. These methods will henceforth be referred using their corresponding numbers in the paper.

In Fig. 5, an angiography image is considered and the goal is to extract the blood vessels. The number of occurrences of a few gray values are very large compared to the others in the histogram of this image. As can be seen from the figure, the proposed methodology outperforms the others as it extracts out the thinner blood vessels successfully. An image of a galaxy is considered in Fig. 6. The graylevel histogram of this image is almost unimodal in nature and, hence, extracting multiple regions from it is a nontrivial task. We use the proposed multilevel thresholding scheme and the various other schemes to find out the total extent and the core region of the galaxy. It is evident from the figure that the results obtained using the proposed methodology is as good as or better than the others.

Let us now consider the quantitative evaluation of segmentation results. Table I lists the quantitative measures of object/ background separation and segmentation performance of the various thresholding algorithms. The serial numbers in the first (left most) column of the table represent various images used, whereas those in the first (top most) row represent the techniques considered. Object/background separation has been carried out on the first five images [images (1)-(5)] and multiple region segmentation has been performed on the rest of the images.

In the evaluation on the basis on the proposed VMAD-index and $\beta$-index, a higher value indicates better object/background separation or segmentation. Note that both these measures are based on the intra-region homogeneity and the homogeneity of the whole image. Although our methodology [techniques (i) 


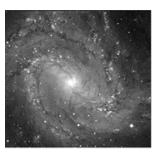

(a)

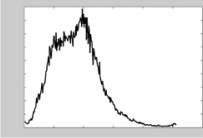

(b)

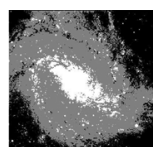

(c)

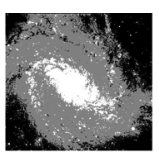

(d)

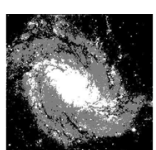

(e)

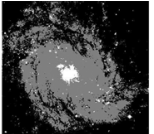

(f)

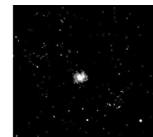

(g)

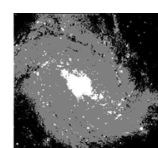

(h)

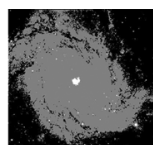

(i)

Fig. 6. Qualitative performance of the various thresholding algorithms applied to find the core and extent of the galaxy in an image. (a) Image. (b) Histogram. (c) Seg. by (i). (d) Seg. by (ii). (e) Seg. by (iii). (f) Seg. by (iv). (g) Seg. by (v). (h) Seg. by (vi). (i) Seg. by (viii).

TABLE I

Quantitative Results ObTained Using the Various Thresholding TeChNiQues on DifFERENT IMAges

\begin{tabular}{|c|c|c|c|c|c|c|c|c|c|}
\hline Images & Techniques: & (i) & (ii) & (iii) & (iv) & (v) & (vi) & (vii) & (viii) \\
\hline \hline \multirow{2}{*}{$(1)$} & $\beta$-index & 2.46 & 2.56 & 2.96 & 2.06 & 1.20 & 2.84 & 1.20 & 2.75 \\
\cline { 2 - 11 } & VMAD-index & 1.11 & 1.19 & 1.99 & 0.97 & 0.96 & 1.70 & 0.96 & 1.96 \\
\hline \multirow{2}{*}{$(2)$} & $\beta$-index & 5.65 & 8.71 & 15.18 & 1.001 & 5.862 & 14.47 & 8.25 & 11.92 \\
\cline { 2 - 11 } & VMAD-index & 5.65 & 4.05 & 3.04 & 0.998 & 2.37 & 3.86 & 2.9 & 2.96 \\
\hline \multirow{2}{*}{$(3)$} & $\beta$-index & 4.83 & 5.73 & 6.68 & 1.7 & 5.3 & 3.88 & 1.09 & 6.49 \\
\cline { 2 - 10 } & VMAD-index & 9.17 & 9.91 & 8.37 & 1.11 & 7.01 & 9.79 & 0.992 & 8.16 \\
\hline \multirow{2}{*}{$(4)$} & $\beta$-index & 3.4 & 4.32 & 6.49 & 1.03 & 3.28 & 5.43 & 1.69 & 6.32 \\
\cline { 2 - 10 } & VMAD-index & 1.62 & 2.43 & 2.26 & 0.979 & 1.84 & 2.53 & 0.935 & 2.15 \\
\hline \multirow{2}{*}{$(5)$} & $\beta$-index & 2.3 & 2.31 & 2.32 & 1.69 & 1.44 & 2.29 & 1.13 & 1.02 \\
\cline { 2 - 10 } & VMAD-index & 1.7 & 1.66 & 1.6 & 1.08 & 1.07 & 1.53 & 1.003 & 0.991 \\
\hline \multirow{2}{*}{$(6)$} & $\beta$-index & 12.07 & 3.055 & 12.23 & 10.6 & 2.082 & 10.29 & n.a. & 2.4 \\
\cline { 2 - 10 } & VMAD-index & 0.12 & 0.18 & 0.119 & 0.121 & 0.144 & 0.117 & n.a. & 0.214 \\
\hline \multirow{2}{*}{$(7)$} & $\beta$-index & 11.34 & 12.22 & 12.69 & 9.958 & 9.368 & 7.644 & n.a. & 11.56 \\
\cline { 2 - 10 } & VMAD-index & 3.7 & 3.47 & 2.96 & 5.72 & 5.67 & 5.79 & n.a. & 3.57 \\
\hline \multirow{2}{*}{$(8)$} & $\beta$-index & 4.56 & 4.7 & 4.79 & 3.85 & 1.18 & 4.05 & n.a. & 2.89 \\
\cline { 2 - 10 } & VMAD-index & 1.58 & 1.64 & 1.79 & 1.65 & 0.984 & 1.57 & n.a. & 1.76 \\
\hline
\end{tabular}

and (ii)] is based on the calculation of certain association errors which do not use any image homogeneity measure, the performance is comparable to the others in terms of both VMADindex and $\beta$-index. Moreover, it is evident from the table that if we consider the evaluation only in terms of the proposed VMAD-index, our techniques outperform most of the others.

We find that the proposed VMAD-index relates more to the visual observation of the segmentation results. For example, consider the image (2) in Table I. It is the image which is considered in Fig. 5. The VMAD-index like the qualitative results suggests that the proposed methodology performs better than the others, whereas the $\beta$-index does not. This is due to the fact that the $\beta$-index measure is influenced by outliers unlike the VMAD-index.

Note that as described in Section III, two gray values $g_{d}$ and $g_{b}$ are needed to be predefined in order to use the proposed thresholding methodology. We have considered (for 256-level grayscale images) $g_{d}=g_{\min }+20$ and $g_{b}=g_{\max }-20$.

\section{B. Edge Extraction}

We present here the use of the proposed thresholding methodology in carrying out edge extraction from images of gradient magnitudes, referred to as gradient images. It is mentioned in [17] that quantitative evaluation of edge detection, which does not use ground truth, is often misleading especially when used on images with complex scenes. As our prime goal is to compare the proposed thresholding methodology to others, we shall use only qualitative evaluation to judge the performance of edge extraction by the various techniques.

Gradient magnitude histograms are in general unimodal and positively (right) skewed in nature. In literature, very few techniques have been proposed to carry out bilevel thresholding in such histograms. Among these techniques, we consider the following for comparison: (ix) unimodal histogram thresholding technique by Rosin [6] and (x) the thresholding technique by
Henstock et al. [7]. In addition to the aforesaid techniques, we also consider here some of the existing thresholding techniques mentioned previously in this section.

As mentioned earlier in this section and in Section III, two gray values $g_{d}$ and $g_{b}$ are needed as input parameters in order to use the proposed thresholding methodology. While using the proposed thresholding methodology on gradient images, $g_{d}$ and $g_{b}$ represent two gradient magnitude values and we consider the input parameters as $g_{d}=g_{\min }+\max \left(\left[10 g_{3 \%}\right]\right)$ and $g_{b}=$ $g_{\max }-\max \left(\left[10 g_{97 \%}\right]\right)$. The notation $g_{\rho \%}$ denotes the $\rho$ th percentile of the gradient magnitude in the distribution (gradient magnitude histogram).

Figs. 7 and 8 give the qualitative evaluation of the edge extraction performance of the various thresholding algorithms. In Fig. 7, we find that the proposed technique does much better than the others in determining the valid edges and eliminating those due to the inherent noise and texture. In Fig. 8, we find three regions in the gradient image. One (white) represents the gradient values which surely correspond to valid edges, another (black) represents those which surely do not correspond to valid edges and the third region (gray) represents the gradient values which could possibly correspond to valid edges. Such multilevel thresholding in gradient magnitude histograms could be used along with the hysteresis technique suggested in [18] in order to determine the actual edges. We see from the figure that the proposed techniques perform as good as or better than the others. Note that, edge thinning has not been done in the results shown in Figs. 7 and 8, as it is not of much significance with respect to the intended comparisons.

\section{Parameter Dependency of the Proposed Methodology}

As given in Section III, the proposed bilevel thresholding methodology requires two input parameters, namely, $g_{d}$ and $g_{b}$, which give the range of element values belonging to the initial regions. The performance analysis of the proposed methodology 


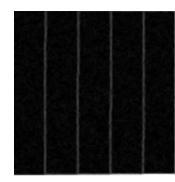

(a)

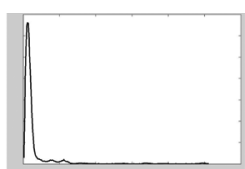

(b)

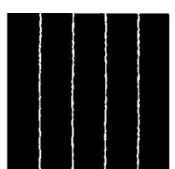

(c)

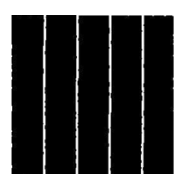

(d)

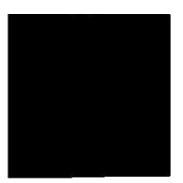

(e)

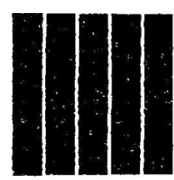

(f)

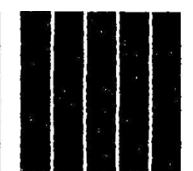

(g)

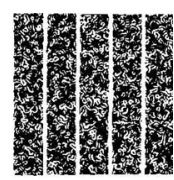

(h)

Fig. 7. Qualitative performance of the various thresholding algorithms applied to mark the edges in a gradient image. (a) Image. (b) Histogram. (c) Edges by (i) (d) Edges by (ii). (e) Edges by (iii). (f) Edges by (vii). (g) Edges by (ix). (h) Edges by (x).

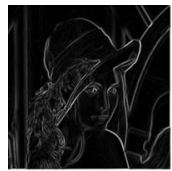

(a)

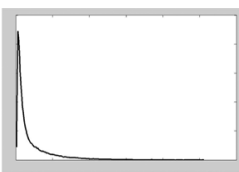

(b)

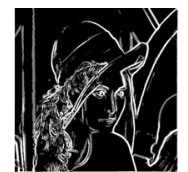

(c)

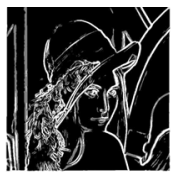

(d)

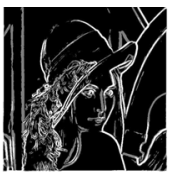

(e)

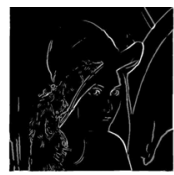

(f)

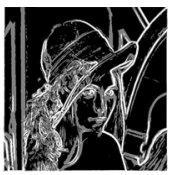

(g)

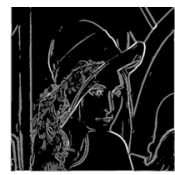

(h)

Fig. 8. Qualitative performance of the various thresholding algorithms applied to obtain the edge, nonedge and possible edge regions in a gradient image. (a) Image. (b) Histogram. (c) Edges by (i). (d) Edges by (ii). (e) Edges by (iii). (f) Edges by (iv). (g) Edges by (v). (h) Edges by (vi).

TABLE II

Parameter Dependency Analysis of the Proposed Fuzzy (F) And Rough (R) Set Theory Based Thresholding Methods

\begin{tabular}{|c|c|c|c|c|c|c|c|c|c|c|c|}
\hline \multicolumn{2}{|c|}{ Parameter Dependence } & II & 12 & 13 & 14 & 15 & 16 & 17 & 18 & 19 & 110 \\
\hline \multirow{2}{*}{\multicolumn{2}{|c|}{$\begin{array}{l}\text { MAD1 (F) } \\
\text { MAD2 (F) }\end{array}$}} & 2.533 & 5.281 & 6.865 & 10.381 & $\begin{array}{ll}1.87 \\
\end{array}$ & 4.298 & 6.58 & 6.867 & $\begin{array}{ll}7.721 \\
\end{array}$ & 5.535 \\
\hline & & 1.85 & 4.6 & 5.595 & 9.73 & 1.755 & 3.335 & 5.69 & 6.345 & 5.61 & 5.755 \\
\hline \multirow{2}{*}{\multicolumn{2}{|c|}{$\begin{array}{l}\text { MAD1 (R) } \\
\text { MAD2 (R) }\end{array}$}} & 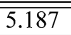 & 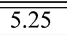 & $\overline{5.125}$ & 7.062 & 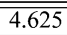 & 7.25 & $\overline{7}$ & $\begin{array}{ll}6.328 \\
\end{array}$ & 5.46 & 5.625 \\
\hline & & 6 & 5 & 4 & 6 & 4 & 8 & 7 & 5.5 & 5 & 6 \\
\hline Parameter & I11 & $\mathrm{I} 12$ & I13 & $\mathrm{I} 14$ & $\mathrm{I} 15$ & Mean & $\begin{array}{l}\text { Standarc } \\
\text { Deviatio }\end{array}$ & \multicolumn{4}{|c|}{\begin{tabular}{|c|} 
Lower and Upper Bound \\
$(99 \%$ confi dence interval $)$
\end{tabular}} \\
\hline MAD1 (F) & 2.58 & 3.263 & 1.512 & 2.742 & (5.813 & $\overline{4.923}$ & 2.525 & & {$[0(-1.581$} & 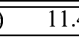 & \\
\hline MAD2 (F) & 1.975 & 3.215 & 1.32 & 2.405 & 5.75 & 4.328 & 2.326 & & {$[0(-1.663$} & & \\
\hline MAD1 (R) & 3.828 & 6.875 & 4.312 & 5 & 6.281 & 5.680 & 1.068 & & {$[2.827$} & 8.33 & \\
\hline MAD2 (R) & 3.5 & 5 & 4.5 & 4 & 5 & 5.233 & 1.222 & & {$[2.083$} & 8.38 & \\
\hline
\end{tabular}

would be incomplete without assessing the dependency of the proposed techniques on these input parameters.

In order to assess the dependency of the algorithms on the input parameters, we consider fifteen different images (I1-I15) and apply the proposed bilevel histogram thresholding techniques on them in order to carry out object/background separation. Sixteen different pairs of input parameters are used to get different threshold values and the variation in the threshold values is then analyzed statistically. Table II lists the mean of absolute deviation from the mean (MAD1) and the median of absolute deviation from the median (MAD2) of the threshold values for each image I1 to I15 obtained using the sixteen different pairs of parameters with the proposed technique of thresholding based on fuzzy (denoted by F) and rough (denoted by R) measure of association errors. Then we present the $99 \%$ confidence interval within which the MAD1 and MAD2 measure would lie, considering the MAD1 and MAD2 measures as values of random variables having normal density functions with their mean and standard deviation as the sample mean and sample standard deviation of the MAD1 and MAD2 values, respectively.

We see that the $99 \%$ confidence intervals of the MAD1 and MAD2 measures for the proposed thresholding technique based on fuzzy measure of association errors are [0 11.428] and [0 10.321], respectively and that of the MAD1 and MAD2 measures for the proposed thresholding technique based on rough measure of association errors are [2.8279 8.3335] and [2.08368.383]. Therefore, we see that, in general, the difference in threshold values obtained using the proposed methodology with different parameters is around 10 , and, hence, the dependency of the techniques on the input parameters is not of much significance.

\section{CONCLUSION}

In this paper, a novel bilevel histogram thresholding methodology has been proposed. Each element of the histogram has been associated with one of the two regions by comparing the corresponding errors due to the associations. The errors due to associations have been obtained using image ambiguity measures given by the linear index of fuzziness or the rough entropy measures. The proposed bilevel thresholding methodology has then been used in a tree structured technique to present a novel multilevel histogram thresholding algorithm.

The proposed methods of bilevel and multilevel thresholding do not make any prior assumptions about the histogram unlike many existing techniques. The proposed methodology has been used to perform segmentation and edge extraction on grayscale and gradient magnitude images, respectively. A new quantitative index called the VMAD-index has also been proposed to evaluate image segmentation performance. The VMAD-index has been based on the median of absolute deviation from median measure, which is a well-known robust estimator of scale.

Qualitative and quantitative performance of the proposed methodology have been studied and compared to that of a few existing thresholding techniques. The methods based on measures of association error have been found suitable for images with different kinds of histograms. Extensive experimental results have suggested that the proposed techniques perform as good as or better than the others.

\section{REFERENCES}

[1] N. Otsu, "A threshold selection method from gray-level histogram," IEEE Trans. Syst., Man, Cybern., vol. SMC-9, no. 1, pp. 62-66, Jan. 1979. 
[2] J. N. Kapur, P. K. Sahoo, A. K. C. Wong, and C. Wong, "A new method for gray-level picture thresholding using the entropy of the histogram," Comput. Vis., Graph., Image Process., vol. 29, pp. 273-285, 1985.

[3] J. Kittler and J. Illingworth, "Minimum error thresholding," Pattern Recognit., vol. 19, no. 1, pp. 41-47, 1986.

[4] S. K. Pal, R. A. King, and A. A. Hashim, "Automatic grey level thresholding through index of fuzziness and entropy," Pattern Recognit. Lett., vol. 1, no. 3, pp. 141-146, 1983.

[5] W.-H. Tsai, "Moment-preserving thresholding: A new approach," Comput. Vis., Graph., Image Process., vol. 29, pp. 377-393, 1985.

[6] P. L. Rosin, "Unimodal thresholding," Pattern Recognit., vol. 34, no. 11, pp. 2083-2096, 2001.

[7] P. V. Henstock and D. M. Chelberg, "Automatic gradient threshold determination for edge detection," IEEE Trans. Image Process., vol. 5, no. 5, pp. 784-787, May 1996.

[8] J.-S. Lee, M. C. K. Yang, and K. Yang, "Threshold selection using estimates from truncated normal distribution," IEEE Trans. Syst., Man, Cybern., vol. 19, no. 2, pp. 422-429, Feb. 1989.

[9] M. Sezgin and B. Sankur, "Survey over image thresholding techniques and quantitative performance evaluation," J. Electron. Imag., vol. 13, no. 1, pp. 146-168, 2004.

[10] P. K. Sahoo, S. Soltani, A. K. C. Wong, and Y. C. Chen, "A survey of thresholding techniques," Comput. Vis. Graph. Image Process., vol. 41, no. 2, pp. 233-260, 1988.

[11] O. J. Tobias and R. Seara, "Image segmentation by histogram thresholding using fuzzy sets," IEEE Trans. Image Process., vol. 11, no. 12, pp. 1457-1465, Dec. 2002.

[12] D. Sen and S. K. Pal, "Histogram thresholding using beam theory and ambiguity measures," Fundam. Inf., vol. 75, no. 1-4, pp. 483-504, 2007.

[13] F. Hampel, "The influence curve and its role in robust estimation," $J$. Amer. Statist. Assoc., vol. 69, no. 346, pp. 383-393, 1974.

[14] L. Breiman, J. H. Friedman, R. A. Olshen, and C. J. Stone, Classification and Regression Trees. Boca Raton, FL: CRC, 1998.

[15] S. K. Pal, A. Ghosh, and B. U. Shankar, "Segmentation of remotely sensed images with fuzzy thresholding, and quantitative evaluation," Int. J. Remote Sens., vol. 21, no. 11, pp. 2269-2300, 2000.

[16] P. J. Rousseeuw and C. Croux, "Alternatives to the median absolute deviation,” J. Amer. Statist. Assoc., vol. 88, no. 424, pp. 1273-1283, 1993.

[17] M. Heath, S. Sarkar, T. Sanocki, and K. Bowyer, "A robust visual method for assessing the relative performance of edge-detection algorithms," IEEE Trans. Pattern Anal. Mach. Intell., vol. 19, no. 12, pp. 1338-1359, Dec. 1997.

[18] J. Canny, "A computational approach to edge detection," IEEE Trans. Pattern Anal. Mach. Intell., vol. PAMI-8, no. 6, pp. 679-698, Jun. 1986.

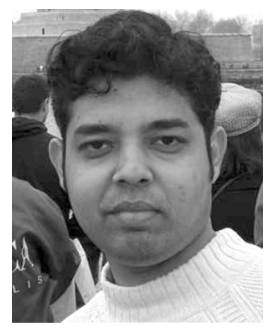

Debashis Sen (S'05-M'06) received B.Eng. (Hons.) degree in electronics and communication engineering from the University of Madras, Chennai, India, in 2002, and the M.A.Sc. degree in electrical engineering from Concordia University, Montreal, QC, Canada, in 2005. He is currently pursuing the $\mathrm{Ph} . \mathrm{D}$. degree in computer engineering at the Center for Soft Computing Research, Indian Statistical Institute, Kolkata, India.

He was with the Center for Signal Processing and Communications and the Video Processing and Communications Group, Concordia University, from 2003-2005. His research interests include image and video processing, probability theory, and soft computing.

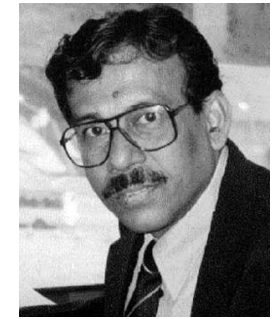

Sankar K. Pal (M'81-SM'84-F'93) received the $\mathrm{Ph} . \mathrm{D}$. degree in radio physics and electronics from the University of Calcutta, India, in 1979, and the $\mathrm{Ph} . \mathrm{D}$. degree in electrical engineering along with DIC from Imperial College, University of London, London, U.K., in 1982.

$\mathrm{He}$ is the Director and Distinguished Scientist of the Indian Statistical Institute, Kolkata. Currently, he is a J. C. Bose Fellow of the Government of India. He founded the Machine Intelligence Unit and the Center for Soft Computing Research, a National Facility in the Institute in Calcutta. He was with the University of California, Berkeley, and the University of Maryland, College Park, from 1986-1987; the NASA Johnson Space Center, Houston, TX, from 1990-1992 and 1994; and the U.S. Naval Research Laboratory, Washington, DC, in 2004.

Prof. Pal is a Fellow of the Academy of Sciences for the Developing World (TWAS), Italy, International Association for Pattern recognition, USA, International Association of Fuzzy Systems, USA, and all four National Academies for Science/Engineering in India. Since 1997, he has served as a Distinguished Visitor of IEEE Computer Society (USA) for the Asia-Pacific Region and has held several visiting positions in Hong Kong and Australian universities. He is a coauthor of fourteen books and more than three hundred research publications in the areas of pattern recognition and machine learning, image processing, data mining and web intelligence, soft computing, neural nets, genetic algorithms, fuzzy sets, rough sets and bioinformatics. He received the $1990 \mathrm{~S}$. S. Bhatnagar Prize (which is the most coveted award for a scientist in India), and many prestigious awards in India and abroad, including the 1999 G. D. Birla Award, the 1998 Om Bhasin Award, the 1993 Jawaharlal Nehru Fellowship, the 2000 Khwarizmi International Award from the Islamic Republic of Iran, the 2000-2001 FICCI Award, the 1993 Vikram Sarabhai Research Award, the 1993 NASA Tech Brief Award (USA), the 1994 IEEE TRANSACTIONS ON NeURAL NeTwORKS Outstanding Paper Award, the 1995 NASA Patent Application Award (USA), the 1997 IETE-R. L. Wadhwa Gold Medal, the 2001 INSA-S. H. Zaheer Medal, the 2005-2006 ISC-P. C. Mahalanobis Birth Centenary Award (Gold Medal) for Lifetime Achievement, the 2007 J. C. Bose Fellowship of the Government of India, and the 2008 Vigyan Ratna Award from the Science and Culture Organization, West Bengal. He was an Associate Editor of IEEE TRANSACTIONS ON PATTERN ANALYSIS AND MaChINE INTELLIGENCE (2002-2006), the IEEE TRANSACTIONS ON NEURAL NETwORKS (1994-1998 and 2003-2006), Neurocomputing (1995-2005), Pattern Recognition Letters, International Journal of Pattern Recognition and Artificial Intelligence, Applied Intelligence, Information Sciences, Fuzzy Sets and Systems, Fundamenta Informaticae, LNCS Transactions on Rough Sets, International Journal of Computational Intelligence and Applications, IET Image Processing, Journal Intelligent Information Systems, and Proc. INSA-A. He is a Book Series Editor of Frontiers in Artificial Intelligence and Applications (IOS Press) and Statistical Science and Interdisciplinary Research (World Scientific). He is a Member of the Executive Advisory Editorial Board, IEEE TRANSACTIONS ON FUZZY SysteMs, International Journal on Image and Graphics, and International Journal of Approximate Reasoning; , and a Guest Editor of IEEE Computer. 